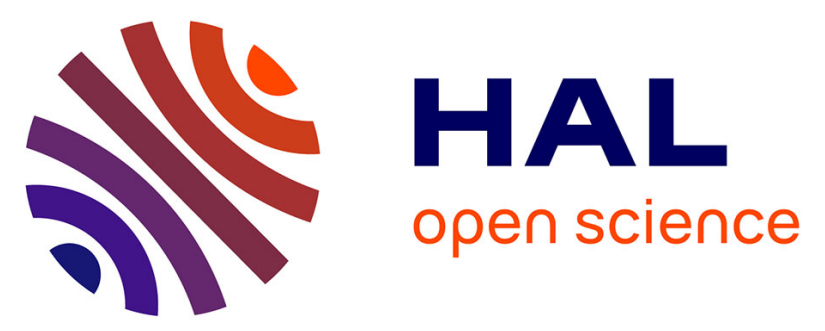

\title{
Computation of the relaxation effective moduli for fibrous viscoelastic composites using the asymptotic homogenization method
}

R. Rodríguez-Ramos, J.A. Otero, O.L. Cruz-González, R. Guinovart-Díaz, J. Bravo-Castillero, F.J. Sabina, P. Padilla, Frédéric Lebon, I. Sevostianov

\section{To cite this version:}

R. Rodríguez-Ramos, J.A. Otero, O.L. Cruz-González, R. Guinovart-Díaz, J. Bravo-Castillero, et al.. Computation of the relaxation effective moduli for fibrous viscoelastic composites using the asymptotic homogenization method. International Journal of Solids and Structures, 2020, 190, pp.281-290. 10.1016/j.ijsolstr.2019.11.014 . hal-02909269

\section{HAL Id: hal-02909269 \\ https://hal.science/hal-02909269}

Submitted on 26 Oct 2021

HAL is a multi-disciplinary open access archive for the deposit and dissemination of scientific research documents, whether they are published or not. The documents may come from teaching and research institutions in France or abroad, or from public or private research centers.
L'archive ouverte pluridisciplinaire HAL, est destinée au dépôt et à la diffusion de documents scientifiques de niveau recherche, publiés ou non, émanant des établissements d'enseignement et de recherche français ou étrangers, des laboratoires publics ou privés. 


\title{
Computation of the relaxation effective moduli for fibrous viscoelastic composites using the asymptotic homogenization method
}

\author{
R. Rodríguez-Ramos ${ }^{\mathrm{a}, *}$, J.A. Otero ${ }^{\mathrm{b}}$, O.L. Cruz-González ${ }^{\mathrm{c}}$, R. Guinovart-Díaz ${ }^{\mathrm{a}}$, \\ J. Bravo-Castillero ${ }^{\text {d, F.J. Sabina }}{ }^{\mathrm{d}}$, P. Padilla ${ }^{\mathrm{d}}$, F. Lebon ${ }^{\mathrm{c}}$, I. Sevostianov ${ }^{\mathrm{e}}$ \\ a Facultad de Matemática y Computación, Universidad de La Habana, San Lázaro y L, Vedado, La Habana, CP 10400, Cuba \\ b Departamento de Ciencias, Escuela de Ingeniería y Ciencias, Tecnológico de Monterrey CEM, Atizapán de Zaragoza, EM CP 52926, Mexico \\ c Aix Marseille Univ, CNRS, Centrale Marseille, LMA UMR 7031, Marseille, France \\ d Universidad Nacional Autónoma de México, Instituto de Investigaciones en Matemáticas Aplicadas y en Sistemas, Alcaldía Álvaro Obregón, 01000 CDMX, \\ Mexico \\ e Department of Mechanical and Aerospace Engineering, New Mexico State University, Las Cruces, NM 88003, USA.
}

\begin{abstract}
A two-phase parallel fibre-reinforced periodic viscoelastic composite is considered wherein the constituents are isotropy. Simple closed-form formulae are obtained for the effective properties of composites with square and hexagonal cells by means of the two-scale asymptotic homogenization method. The computation of the effective properties of non-ageing linear viscoelastic composites with periodic structure containing long cylindrical fibres of circular cross-section is performed. The local problems and overall viscoelastic properties are obtained in explicit form using the elastic-viscoelastic correspondence principle and assuming perfect contact conditions at the interface between constituents. Comparison with different viscoelastic models allowing explicit inverse Laplace transforms such as, traditional Maxwell and Kelvin models and Rabotnov-Scott Blair fractional exponential model are shown. The analytical results are verified by comparison with computational ones.
\end{abstract}

\section{Introduction}

Several methods have been developed over the past few decades to theoretically predict the effective mechanical properties of composite materials. For linear viscoelastic fibre-reinforced composites, most of the constitutive models can be classified into two groups.

In the first group, the viscoelastic equations are transformed to the linear elastic regime using the method of elastic-viscoelastic correspondence (Volterra, 1912) and the Laplace-Carson transform. This approach allows to treat boundary value problems for linear non-ageing viscoelastic materials as linear elastic ones written in Laplace-Carson space. Thus, homogenization techniques for viscoelastic composites can be performed analytically by using elastic homogenization solutions and treating them as images in the Laplace-Carson space (see, Hashin, 1966 and Lévesque et al., 2007). The main challenge is to obtain analytical formulas for inverse Laplace transform to write viscoelastic solution in time space (see, Lévesque et al., 2007 and Le et al., 2007). This difficulty can

\footnotetext{
* Corresponding author.

E-mail address: reinaldo@matcom.uh.cu (R. Rodríguez-Ramos).
}

be overcome if the integral operator describing viscoelastic effects contains an exponential kernel (that corresponds to various dashpot-spring models). Such kernels, however, are often not appropriate for matching experimental data.

In order to solve this problem, an alternative methodology has been developed based on experimental measurements and theoretical considerations by Scott Blair and Coppen (1939), Scott Blair and Coppen (1943) and Rabotnov (1948), respectively. Herein, they proposed to use fraction-exponential operators instead of the exponential ones. A detailed description of the approach is given in the books of Rabotnov (1977) and Podlubny (1998). Fractionexponential operators are sufficiently flexible to describe experimental data and, at the same time, allow inverse Laplace transforms in explicit analytical form. In addition, they involve parameters that have clear physical sense. To the best of our knowledge, application of the fraction-exponential operators to heterogeneous materials has been first proposed by Shermergor (1977) and coauthors for layered composites. In Levin and Sevostianov (2005) is used this approach to describe overall viscoelastic behavior of matrix composites. They also introduced creep and relaxation contribution tensors that allow one to describe the effect of inhomogeneities on the overall viscoelastic properties in a unified way and 
thus, to extend each of known micromechanical scheme from elastic materials to viscoelastic ones (Sevostianov and Levin, 2015). The concept of creep and relaxation contribution tensor has been used by Sevostianov et al. (2016) to evaluate effective properties of fibre reinforced composites and by Vilchevskaya et al. (2019) to describe replacement relations in viscoelastic heterogeneous materials.

In the second group, the homogenization method is applied directly in the time domain to obtain the constitutive models. The time domain solution may be also obtained with numerical inversion algorithms, such as the collocation method (Schapery, 1964) or quasi-elastic approximation in the Laplace-Carson space (Brenner et al., 2002). This method is computationally-efficient but at the expense of accuracy. Another approach consists of a timeintegration approach, relying on variational principles (Lahellec and Suquet, 2007). While it avoids the Laplace-Carson transforms and solves the viscoelastic problem directly in the time domain, its numerical implementation is challenging.

Different contributions developed for linear viscoelastic composites have been introduced in several recent papers. For example, the authors in Wang and Pindera (2016) extend the elasticitybased, locally-exact homogenization theory for periodic materials with hexagonal and tetragonal symmetries to viscoelastic unidirectional composites. In addition, a spatial-temporal nonlocal homogenization model for transient anti-plane shear wave propagation in viscoelastic composites is presented in Hu and Oskay (2018). Another fruitful research approach is the variational framework developed in Tressou et al. (2018), which focus its attention on the incremental variational approach commonly called EIV approach in the literature proposed by Lahellec and Suquet (2007) for dealing with the linear viscoelastic behavior of different types of microstructures. Also, solutions based on Fourier series analysis are studied in To et al. (2017).

This work has some differences with respect to recently contributions already presented in the literature, see e.g. Yi et al. (1998), Tran et al. (2011), Liu et al. (2004), Cai and Sun (2013), Daridon et al. (2016), Tang and Felicelli (2015). In the aforementioned works, the main relevant issues are summarized as follows: a way to obtain the effective viscoelastic moduli both in the time and frequency domain for viscoelastic composites with periodic microstructures is formulated in Yi et al. (1998) by using the asymptotic homogenization method; Tran et al. (2011) proposed a numerical multiscale method computing the response of structures made of linearly non-aging viscoelastic in the time domain based on RVE method; Liu et al. (2004) predicted the viscoelastic properties of layered materials and obtained explicit formulas for predicting the viscoelastic relaxation modulus of layered materials; (Cai and Sun, 2013) works with four-step three-dimensionally (3D) braided composites; Daridon et al. (2016) deals with imperfect (Kelvin-Voigt) viscoelastic interphases and Tang and Felicelli (2015) proposes an homogenization methodology based on the variational asymptotic method for the unit cell (VAMUCH), where the predictions of effective stress relaxation stiffness are obtained in the time domain without applying the Laplace transform. However, in the present work, the two-scale asymptotic homogenization method initially developed in Guinovart-Díaz et al. (2001), Rodríguez-Ramos et al. (2001) for calculating the effective properties of elastic fibrous composites is extended and generalized to a non-ageing linear viscoelasticity framework. The investigation of the effective properties is based on the correspondence principle and the Laplace transform. The numerical algorithm proposed by Hollenbeck (1998) to invert the Laplace transform is used to calculate the properties of the homogenized composites in the time domain. Consequently, the present formulation contributes with analytical solutions, in the Laplace-Carson space, for the local problems and the effective coefficients for fibrous viscoelastic composites with square and hexagonal cells; closed form expressions eas- ily for their computation are ensured; moreover the behavior of the effective coefficients for square and hexagonal cells for different relaxation kernels as Maxwell-Kelvin model, the fractionexponential function or Rabotnov's kernel and the Kelvin model are analyzed; finally, numerical results and comparisons with other models are provided as validation of the proposed approach.

\section{Viscoelastic heterogeneous problem. Basic equations}

Let us denote by $\Omega \in \mathbb{R}^{3}$ a linear viscoelastic composite material with periodic structure and cylindrical cross-section fibres (see Fig. 1 (a)). The domain $\Omega$ is constituted by two phases: the matrix $\Omega_{m}^{\xi}$ and the finite collection of disjoints cylindrical cross-section fibres $\Omega_{f}^{\xi}$, such that $\bar{\Omega}=\bar{\Omega}_{m}^{\xi} \cup \bar{\Omega}_{f}^{\xi}$ and $\Omega_{m}^{\xi} \cap \Omega_{f}^{\xi}=\emptyset$. The fibres are oriented along the $\mathrm{Ox}_{3}$-axis. The interface between the two constituents is denoted by $\Gamma^{\xi}$ (see Fig. 1(b)). The fibres are embedded into the matrix within the unit cell, which can be square or hexagonal. Besides, the unitary periodic cell is denoted by $Y$ (see Fig. 1(c)).

Then, ignoring inertia term, the equilibrium equation under the action of external volume forces is written as,

$$
\begin{aligned}
& \nabla \cdot \boldsymbol{\sigma}(\boldsymbol{x}, t)+\boldsymbol{f}(\boldsymbol{x}, t)=\mathbf{0} \quad \text { in }\left(\Omega \backslash \Gamma^{\xi}\right) \times \mathbb{R} \\
& \boldsymbol{u}(\boldsymbol{x}, t)=\overline{\boldsymbol{u}} \quad \text { on } \Omega_{\mathrm{d}} \times \mathbb{R} \\
& \boldsymbol{\sigma}(\boldsymbol{x}, t) \cdot \boldsymbol{n}=\overline{\boldsymbol{S}} \quad \text { on } \partial \Omega_{n} \times \mathbb{R}
\end{aligned}
$$

$\boldsymbol{u}(\boldsymbol{x}, t)=\mathbf{0} \quad$ in $\Omega \times\{0\}$

where $\boldsymbol{\sigma}\left(\sigma_{i j}\right)$ represents the second-order stress tensor, $\boldsymbol{u}\left(u_{i}\right)$ is the viscoelastic displacement, $\boldsymbol{x}\left(x_{i}\right)$ denotes the global Cartesian coordinates, $\boldsymbol{f}\left(f_{i}\right)$ represents the action of external volume forces and satisfies $\boldsymbol{f}(\boldsymbol{x}, t) \in L^{2}(\Omega \times \mathbb{R}), \overline{\boldsymbol{u}}\left(\bar{u}_{i}\right)$ and $\overline{\boldsymbol{S}}\left(\bar{S}_{i}\right)$ are the prescribed displacement and traction on the boundary $\partial \Omega=\partial \Omega_{d} \cup$ $\partial \Omega_{n}$, with $\partial \Omega_{d} \cap \partial \Omega_{n}=\emptyset, \partial \Omega_{d}$ is a part of the surface $\partial \Omega$ where the displacement is prescribed and $\boldsymbol{n}\left(n_{i}\right)$ is the outward unit vector normal to the surface $\partial \Omega$.

In the process of two scales asymptotic homogenization method, it is natural to use $L$ as the characteristic length of the heterogeneous medium and $l$ referring to the length of the periodic cell, such that $l \ll L$. In addition, a small geometric parameter $\xi=l / L$ with $\xi \ll 1$ is introduced. Therefore, the macro or global variable $\boldsymbol{x}\left(x_{i}\right)$ and micro or local variable $\boldsymbol{y}\left(y_{i}\right)$ are related to $\boldsymbol{y}=\boldsymbol{x} / \xi$.

The viscoelastic stress and strain fields are linearly related by the constitutive law (see, Christensen, 1982 and Pipkin, 1986),

$\boldsymbol{\sigma}(\boldsymbol{x}, t)=\int_{0}^{t} \boldsymbol{R}(\boldsymbol{y}, t-\tau): \frac{\partial \boldsymbol{\varepsilon}(\boldsymbol{u}(\boldsymbol{x}, \tau))}{\partial \tau} d \tau$,

where $\boldsymbol{R}\left(R_{i j k l}\right)$ denotes the relaxation modulus and it fulfills the symmetry properties $R_{i j k l}=R_{j i k l}=R_{i j l k}=R_{k l i j}$. We assume $R \in$ $L^{\infty}(\Omega \times \mathbb{R})$, also that it is positively defined and Y-periodic related to the variable $\mathrm{y}$. In addition, the second-order strain tensor is referred to as $\varepsilon\left(\varepsilon_{k l}\right)$ in components.

The following relationship is ensured for small displacements

$\varepsilon_{k l}(\boldsymbol{u}(\boldsymbol{x}, t))=\frac{1}{2}\left(\frac{\partial u_{k}(\boldsymbol{x}, t)}{\partial x_{l}}+\frac{\partial u_{l}(\boldsymbol{x}, t)}{\partial x_{k}}\right)$.

The statement of the constitutive law (2) corresponds to the special form of non-ageing linear viscoelastic materials (Maghous and Creus, 2003). Therefore, the problem (1a)-(2) can be transformed into an elastic one using the Laplace-Carson transform. The aforementioned is known as the correspondence principle. Thus, 
$\Omega$

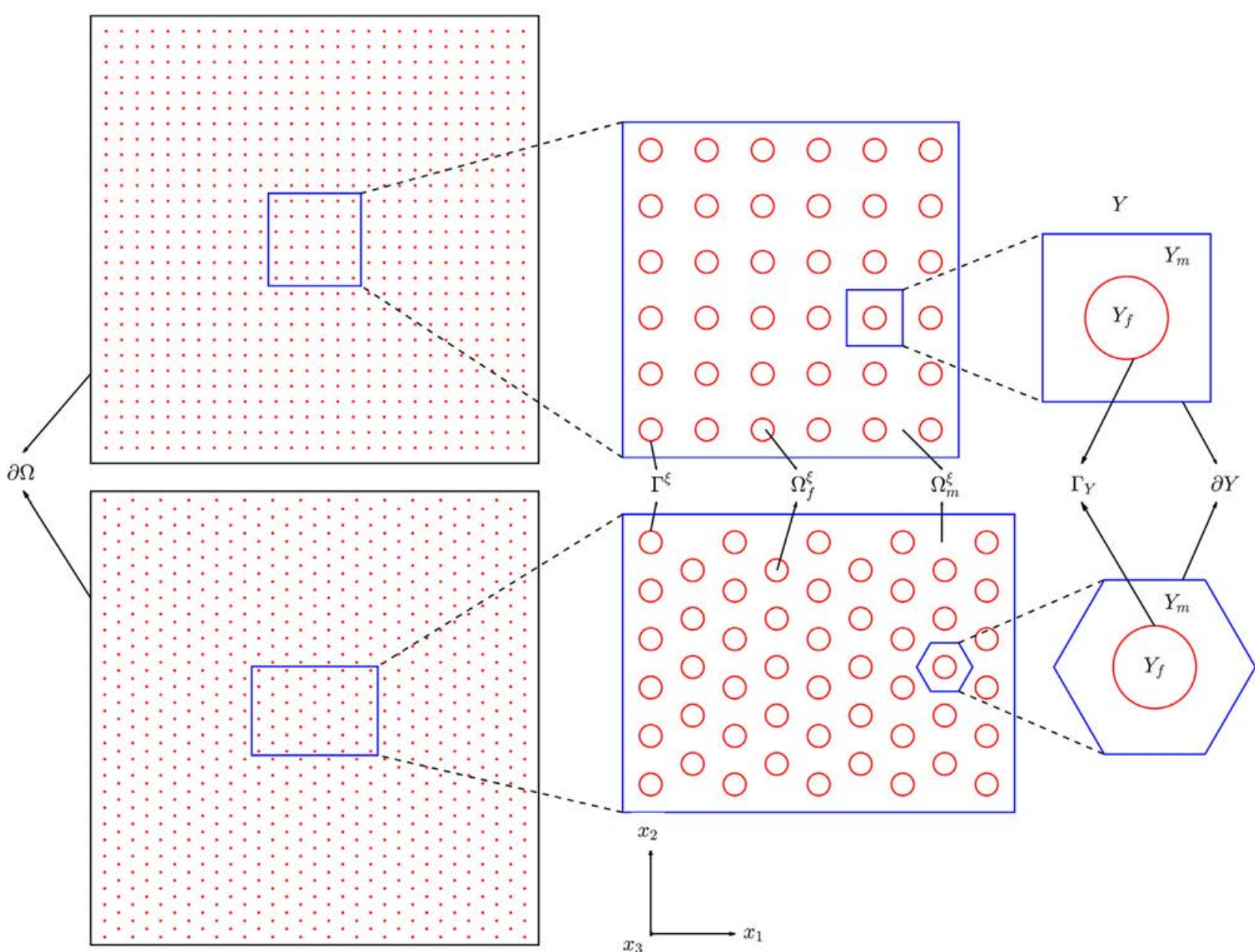

(a)

(b)

(c)

Fig. 1. Macroscopic heterogeneous structure with square and hexagonal cells.

applying the Laplace-Carson transform to (1a)-(2), the mathematical expression for the linear viscoelastic heterogeneous problems in the Laplace-Carson space is written,

$\frac{\partial}{\partial x_{j}}\left[\hat{R}_{i j k l}(\boldsymbol{y}, s) \xi_{k l}(\hat{\boldsymbol{u}}(\boldsymbol{x}, s))\right]+\hat{f}_{i}(\boldsymbol{x}, s)=0, \quad$ in $\left(\Omega \backslash \Gamma^{\xi}\right) \times[0,+\infty)$.

where $\xi_{k l}$ denotes de strain (3) in the Laplace-Carson space. The corresponding boundary conditions associated to (4) are

$\hat{u}_{i}(\boldsymbol{x}, s)=\bar{u}_{i}, \quad$ on $\partial \Omega_{d} \times[0,+\infty)$,

$\hat{R}_{i j k l}(\boldsymbol{y}, s) \xi_{k l}(\hat{\boldsymbol{u}}(\boldsymbol{x}, s)) n_{j}=\bar{S}_{i}, \quad$ on $\partial \Omega_{n} \times[0,+\infty)$,

and the initial condition is taken as follows

$\hat{u}_{i}(\boldsymbol{x}, s)=0, \quad$ in $\Omega \times\{0\}$.

From now on, the functions with the symbol () depending on the parameter $s$ denote corresponding the Laplace-Carson transform.

\section{Two-scale asymptotic homogenization method applied to heterogeneous problem}

The aim of this section is to solve the heterogeneous problem (4)-(7) using AHM.

A formal asymptotic solution for the problem is proposed as follows,

$\hat{\boldsymbol{u}}(\boldsymbol{x}, \xi, s)=\sum_{a=0}^{+\infty} \xi^{a} \hat{\boldsymbol{u}}^{(a)}(\boldsymbol{x}, \boldsymbol{y}, s)$,

where $\hat{\boldsymbol{u}}^{(a)}\left(\hat{u}_{i}^{(a)}\right)$ is Y-periodic related to the variable $\boldsymbol{y} \forall a, \forall \boldsymbol{x} \in$ $\Omega, \forall s \in[0,+\infty)$ and $\hat{\boldsymbol{u}}^{(a)}(\boldsymbol{x}, \boldsymbol{y}, s) \in C^{\infty}\left(\Omega \times \mathbb{R}^{3} \times[0,+\infty)\right)$.

As the material property is periodic in $y$ then, according to the chain rule, the derivative in relation to the global coordinate yields the transformation

$\frac{\partial(\cdot)}{\partial x_{j}}=\frac{\partial(\cdot)}{\partial x_{j}}+\frac{1}{\xi} \frac{\partial(\cdot)}{\partial y_{j}}$.

Using a similar idea (9), the Eq. (3) applied to an arbitrary function of the form $\hat{\boldsymbol{\Phi}}(\boldsymbol{x}, \boldsymbol{x} / \xi, s)$ becomes,

$\varepsilon_{k l}(\hat{\boldsymbol{\Phi}}(\boldsymbol{x}, \boldsymbol{x} / \xi, s))=\varepsilon_{k l x}(\hat{\boldsymbol{\Phi}}(\boldsymbol{x}, \boldsymbol{y}, s))+\xi^{-1} \varepsilon_{k l y}(\hat{\boldsymbol{\Phi}}(\boldsymbol{x}, \boldsymbol{y}, s))$, 
where the expressions $\varepsilon_{k l x}$ and $\varepsilon_{k l y}$ are defined as follows Persson et al. (1993),

$\varepsilon_{k l x}(\hat{\boldsymbol{\Phi}}(\boldsymbol{x}, s))=\frac{1}{2}\left(\frac{\partial \hat{\Phi}_{k}(\boldsymbol{x}, s)}{\partial x_{l}}+\frac{\partial \hat{\Phi}_{l}(\boldsymbol{x}, s)}{\partial x_{k}}\right)$,

and

$\varepsilon_{k l y}(\hat{\boldsymbol{\Phi}}(\boldsymbol{y}, s))=\frac{1}{2}\left(\frac{\partial \hat{\Phi}_{k}(\boldsymbol{y}, s)}{\partial y_{l}}+\frac{\partial \hat{\Phi}_{l}(\boldsymbol{y}, s)}{\partial y_{k}}\right)$.

In this particular case, it is possible to significantly simplify the expressions in the homogenization process using the following operator,

$L_{\alpha \beta}(\bullet):=-\frac{\partial}{\partial \alpha_{j}}\left(\hat{R}_{i j k l}(\boldsymbol{y}, s) \varepsilon_{k l \beta}(\bullet)\right)$,

for $\alpha, \beta=\boldsymbol{x}, \boldsymbol{y}$, indistinctly.

Replacing (8) into (4), taking into account (9)-(13), after some simplifications and grouping in powers of $\xi$, the following sequence of problems is obtained for different orders of the small parameter,

$\xi^{-2}: \quad L_{y y} \hat{\boldsymbol{u}}^{(0)}(\boldsymbol{x}, \boldsymbol{y}, s)=\mathbf{0}$,

$\xi^{-1}: \quad L_{x y} \hat{\boldsymbol{u}}^{(0)}(\boldsymbol{x}, \boldsymbol{y}, s)+L_{y x} \hat{\boldsymbol{u}}^{(0)}(\boldsymbol{x}, \boldsymbol{y}, s)+L_{y y} \hat{\boldsymbol{u}}^{(1)}(\boldsymbol{x}, \boldsymbol{y}, s)=\mathbf{0}$,

$\xi^{0}: \quad L_{x x} \hat{\boldsymbol{u}}^{(0)}(\boldsymbol{x}, \boldsymbol{y}, s)+L_{x y} \hat{\boldsymbol{u}}^{(1)}(\boldsymbol{x}, \boldsymbol{y}, s)+L_{y x} \hat{\boldsymbol{u}}^{(1)}(\boldsymbol{x}, \boldsymbol{y}, s)$

$$
+L_{y y} \hat{\boldsymbol{u}}^{(2)}(\boldsymbol{x}, \boldsymbol{y}, s)-\boldsymbol{f}(\boldsymbol{x})=\mathbf{0} .
$$

Problems (14)-(16) can be solved recursively form considering the solvability condition presented in the following lemma (see Persson et al., 1993, Bakhvalov and Panasenko, 1989),

Lemma 1. Let $\boldsymbol{F}\left(F_{i}\right)$ be square integrable function over $Y$ and consider the boundary value problem

$L_{y y} \boldsymbol{\Phi}=\boldsymbol{F}$,

where $\Phi$ is $Y$-periodic. Then the following conditions hold,

(i) A Y-periodic solution $\boldsymbol{\Phi}$ exists if and only if $\langle\boldsymbol{F}\rangle=0$.

(ii) If a $Y$-periodic solution $\Phi$ exists, then it is unique up to a constant vector $c$

The proof of this lemma is given in Section 4.3 of Persson et al. (1993). The notation $\langle\cdot\rangle$ defines the cell average operator, i.e., $\langle\boldsymbol{F}\rangle:=\frac{1}{|Y|} \int_{\mathrm{Y}} \boldsymbol{F} d y$, where $|Y|$ represents the periodic cell volume.

In what follows, the main results for each power of $\xi$ are given.

Contribution of order $\xi^{-2}$

The problem (14) has the trivial solution $\hat{\boldsymbol{u}}^{(0)}(\boldsymbol{x}, \boldsymbol{y}, s) \equiv \mathbf{0}$. Therefore, Lemma 1 indicates that $\hat{\boldsymbol{u}}^{(0)}(\boldsymbol{x}, \boldsymbol{y}, s)$ is a solution of (14) if and only if it is constant in relation to the variable $\boldsymbol{y}$. It implies that,

$\hat{\boldsymbol{u}}^{(0)}(\boldsymbol{x}, \boldsymbol{y}, s)=\hat{\boldsymbol{v}}(\boldsymbol{x}, s)$,

where $\hat{\boldsymbol{v}}(\boldsymbol{x}, t)$ is a infinitely differentiable function.

Contribution of order $\xi^{-1}$

Using (17), the first term of (15) is zero, $L_{x y} \hat{\boldsymbol{u}}^{(0)}(\boldsymbol{x}, \boldsymbol{y}, s)=$ $L_{x y} \hat{\boldsymbol{v}}(\boldsymbol{x}, s)=\mathbf{0}$. Therefore, the problem (15) becomes,

$L_{y y} \hat{\boldsymbol{u}}^{(1)}(\boldsymbol{x}, \boldsymbol{y}, s)=-L_{y x} \hat{\boldsymbol{u}}^{(0)}(\boldsymbol{x}, \boldsymbol{y}, s)$,

Applying Lemma 1 on (18), taking into account (17), the divergence theorem and the $Y$-periodicity condition of $\hat{\boldsymbol{R}}(\boldsymbol{y}, s)$, the following result can be verified

$\left\langle-L_{y x} \hat{\boldsymbol{u}}^{(0)}(\boldsymbol{x}, \boldsymbol{y}, s)\right\rangle=0$.
Consequently, the existence and uniqueness of a solution for problem (18) is guaranteed. Applying separation of variables, a general solution for (18) is given by

$\hat{\boldsymbol{u}}^{(1)}(\boldsymbol{x}, \boldsymbol{y}, s)=\hat{\boldsymbol{N}}^{r s}(\boldsymbol{y}, s) \varepsilon_{r s x}(\hat{\boldsymbol{v}}(\boldsymbol{x}, s))$,

where $\hat{\boldsymbol{N}}^{r s}\left(\hat{N}_{i}^{r s}\right)$ is called the local function.

Finally, substituting (17) and (19) into (18) and after some simplifications the local problem in relation to the local function is obtained

$-\frac{\partial}{\partial y_{j}}\left(\hat{R}_{i j k l}(\boldsymbol{y}, s) \varepsilon_{k l y}\left(\hat{\boldsymbol{N}}^{r s}(\boldsymbol{y}, s)\right)\right)=\frac{\partial}{\partial y_{j}}\left(\hat{R}_{i j r s}(\boldsymbol{y}, s)\right)$,

where $\hat{\boldsymbol{N}}^{r s}$ is a $Y$-periodic function.

Contribution of order $\xi^{0}$

The existence and uniqueness of a $Y$-periodic solution for the problem (16) is guaranteed if and only if

$\left\langle\boldsymbol{f}(\boldsymbol{x})-L_{x x} \hat{\boldsymbol{u}}^{(0)}(\boldsymbol{x}, \boldsymbol{y}, s)-L_{x y} \hat{\boldsymbol{u}}^{(1)}(\boldsymbol{x}, \boldsymbol{y}, s)-L_{y x} \hat{\boldsymbol{u}}^{(1)}(\boldsymbol{x}, \boldsymbol{y}, s)\right\rangle=0$.

The functions $\hat{\boldsymbol{R}}(\boldsymbol{y}, s)$ and $\hat{\boldsymbol{N}}^{r s}(\boldsymbol{y}, s)$ are $Y$-periodic, hence by (19) and the divergence theorem it is proved that, $\left\langle L_{y x} \hat{\boldsymbol{u}}^{(1)}(\boldsymbol{x}, \boldsymbol{y}, s)\right\rangle=0$.

Finally, working on (21), the homogenized problem is obtained and it can be written in the form

$-\hat{R}_{i j r s}^{(*)}(s) \frac{\partial}{\partial x_{j}} \varepsilon_{r s x}(\hat{\boldsymbol{v}}(\boldsymbol{x}, s))=f_{i}(\boldsymbol{x})$,

where

$\hat{R}_{i j r s}^{(*)}(s)=\left\langle\hat{R}_{i j r s}(\boldsymbol{y}, s)+\hat{R}_{i j k l}(\boldsymbol{y}, s) \varepsilon_{k l y}\left(\hat{\boldsymbol{N}}^{r s}(\boldsymbol{y}, s)\right)\right\rangle$,

is the effective coefficient.

The boundary conditions for the homogenized problem (22) and (23) are rewritten replacing (8) into (5) and (6), respectively. Applying the average operator, we obtain

$\hat{v}_{i}(\boldsymbol{x}, s)=\bar{u}_{i}, \quad$ on $\partial \Omega_{d} \times[0,+\infty)$,

$\hat{R}_{i j r s}^{(*)}(s) \xi_{k l}(\hat{\boldsymbol{v}}(\boldsymbol{x}, s)) n_{j}=\bar{S}_{i}, \quad$ on $\partial \Omega_{n} \times[0,+\infty)$,

and the initial condition is taken as follows

$\hat{v}_{i}(\boldsymbol{x}, s)=0, \quad$ in $\Omega \times\{0\}$.

Consequently, from the previous steps, a recurrent sequence can be obtained allowing the formulation of the local problems (20) defined on the periodic cell $Y$ and the effective coefficients (23) that do not depend on the small epsilon parameter. Thus, the application of the asymptotic homogenization method generates two types of fundamental problems: a) the determination of the effective coefficients from the solutions of the local problems in which the epsilon parameter does not appear, which constitutes the main objective of the present work and, b) from the homogenized problem, the calculation of the boundary problems associated with concrete contour conditions to determine the displacements, deformations and stresses field in which the epsilon parameter plays an important role using the asymptotic expansion proposed in (8). This topic is outside of this work purpose.

It is well known that due to correspondence principle there is an equivalence between elastic and viscoelastic problems in terms of Laplace transforms. Regarding the homogenization approach, in Chapter 2, paragraph 2 of Bakhvalov and Panasenko (1989) is demonstrated the principal steps in constructing and justifying an asymptotic expansion solution. The accuracy of the asymptotic expansion for the small parameter is illustrated in Table 1 on page. 16 of Bakhvalov and Panasenko (1989) where is 
shown the discrepancies and errors for the order of the expansion $n=0,1,2$ and the small parameter $\xi \ll 1$. Besides, in Chapter 6 , paragraph 2 on page 205 of Bakhvalov and Panasenko (1989), the principle of equivalent homogeneity is asserted and the relationship between local characteristics of heterogeneous medium and those of the homogeneous medium described by the averaged equation is carefully studied and proved. The accuracy between heterogeneous solution can be compared with that obtained by the homogenized model using the estimation $\|u-\hat{v}\|_{L^{2}(\Omega)} \leq$ $C \sqrt{\xi}$, where $C$ is a positive constant independent of the small parameter $\xi$.

\section{Two-phase viscoelastic composite. analytical expressions for effective properties}

The idea in this section is to provide closed formulae analogous to Valdivieso-Mijangos et al. (2002), Sabina et al. (2002) but now for the effective viscoelastic characteristics of composites with square and hexagonal arrangement cells. In this sense, two-phase unidirectional fibre reinforced viscoelastic composites are studied here, which consist of identical circular cylindrical isotropic homogeneous fibres embedded in a isotropic homogeneous matrix.

A Cartesian coordinate system $O x_{1} x_{2} x_{3}$ is employed for the geometrical description of composites. The fibres are assumed infinite in the $\mathrm{Ox}_{3}$ direction and periodically distributed without overlapping in directions parallel to the $O x_{1}$ axis and the lines with slope at an angle $\theta=\pi / a, a=2$ for the squared array and $a=3$ for hexagonal array. $R$ is the radius of the fibre circular cross-section and $V_{2}=\pi R^{2}$ with $0 \leq V_{2} \leq \frac{\pi}{4 \sin \theta}$ is the fibre volume fraction on the square and hexagonal periodic cells (see Fig. 1(b)-(c)). Perfect contact conditions are assumed on the interface between matrix and fibre. The relaxation modulus $\hat{\boldsymbol{R}}(\boldsymbol{x}, s)$ is a periodic function of the coordinate $x_{1}$ and $x_{2}$.

The effective properties of these media via AHM have been investigated by many authors. In Otero et al. (2016) semi-analytical formulae were derived for composites under imperfect contact with different inclusions shapes where AHM and finite element method are combined for the computation of the effective characteristics; AHM is used for obtaining the local problems and the analytical expression of the effective properties and finite element method for solving the corresponding two dimensional local problems. The results of Pobedria (1984) were extended in GuinovartDíaz et al. (2001) (for hexagonal cell) and in Rodríguez-Ramos et al. (2001) (for square cell) to the case of transversely isotropic elastic constituents. The formulae for global properties require numerical solution of a infinite linear system of equations. The formulae involve products of vectors and matrices of infinite order which require to be truncated.

Based on the correspondence principle, and the results obtained in Guinovart-Díaz et al. (2001), Rodríguez-Ramos et al. (2001) and Bravo-Castillero et al. (2012), the effective coefficients for two phase viscoelastic composites with isotropic components can be listed as an extension of previous works in the following form,

$$
\begin{aligned}
\hat{k}(s) & =k_{v}(s)-V_{2}\left(k_{1}(s)-k_{2}(s)\right)^{2} K_{\alpha}(a, s) / m_{1}(s) \\
\hat{l}(s) & =l_{v}(s)-V_{2}\left(k_{1}(s)-k_{2}(s)\right)\left(l_{1}(s)-l_{2}(s)\right) K_{\alpha}(a, s) / m_{1}(s), \\
\hat{n}(s) & =n_{v}(s)-V_{2}\left(l_{1}(s)-l_{2}(s)\right)^{2} K_{\alpha}(a, s) / m_{1}(s) \\
\hat{p}(s) & =p_{1}(s)-2 V_{2} p_{1}(s) P_{\alpha}(a, s) \\
\hat{m}(s) & =m_{1}(s)-V_{2}\left(m_{1}(s)-m_{2}(s)\right) M_{\alpha}(a, s) \\
\hat{m}^{\prime}(s) & =m_{1}(s)-V_{2}\left(m_{1}(s)-m_{2}(s)\right) M_{\alpha}^{\prime}(s)
\end{aligned}
$$

where the index $v$ denotes an average property over the periodic cell, i.e. $k_{v}(s)=V_{1} k_{1}(s)+V_{2} k_{2}(s)$ and $V_{1}+V_{2}=1$. The functions $K_{\alpha}(a, s), P_{\alpha}(a, s), M_{\alpha}(a, s)$ and $M_{\alpha}^{\prime}(s)$ which involve the order of accuracy $\alpha$ are given by

$$
\begin{aligned}
K_{\alpha}(a, s) & =C(s) V_{1}+\frac{\delta_{\alpha 2}(2 a-1)\left(1+\kappa_{1}(s)\right) C(s)^{2} R^{4 a}\left(S_{2 a}\right)^{2}}{B^{-1}(s)+R^{4 a-2} E(a, s)}, \\
P_{\alpha}(a, s) & =\frac{\chi_{p}(s)}{1+V_{2} \chi_{p}(s)-\delta_{\alpha 2}(2 a-1)\left(\chi_{p}(s)^{2} R^{4 a}\left(S_{2 a}\right)^{2}\right.}, \\
M_{\alpha}(a, s) & =\frac{1+\kappa_{1}(s)}{\left(1+\kappa_{1}(s) \chi_{m}(s)\right)\left(1+R^{2} H^{-}(a, s)-\delta_{\alpha 2} I(a, s)\right)}, \\
M_{\alpha}^{\prime}(a, s) & =\frac{1+\kappa_{1}(s)}{\left(1+\kappa_{1}(s) \chi_{m}(s)\right)\left(1+R^{2} H^{+}(a, s)-\delta_{\alpha 2} I^{+}(s)\right)},
\end{aligned}
$$

where $E(a, s)=A(s) B^{-1}(s) r(a)+g(a)+(2 a-1) D(s) R^{2}\left(S_{2 a}\right)^{2}, \delta_{k l}$ is the Kronecker's delta and the expressions involved in the above formulae are written as,

$$
\begin{aligned}
H^{ \pm}(a, s)= & A(s) r_{1}(a, s)+B(s) \pi \kappa_{1} / \sin (\pi / a) \\
& \pm(3-a) B(s)\left(S_{4} / \pi+\pi_{1}\right), \\
I(a, s)= & \left\{\begin{array}{cc}
I^{-}(s) \quad \text { for } a=2, \\
\frac{3 R^{8} B^{2}(s)\left(15 R^{2} S_{6}-4 T^{5}\right)^{2}}{1+100 R^{12} A(s)\left(S_{6}\right)^{2}} \quad \text { for } a=3,
\end{array}\right. \\
I^{ \pm}= & \frac{R^{12}\left(A(s) r_{2} \pm B(s) g_{2}\right)\left(A(s) r_{3} \pm B(s) g_{3}\right)}{1+R^{10} A(s) r_{4} \pm B(s) g_{4}}, \\
r(a)= & c_{4 a-1}^{2 a-1} c_{4 a-1}^{2 a+1} R^{4 a-2}\left(S_{4 a}\right)^{2}, \quad r_{1}(a)=(2 a-1)\left(S_{2 a}\right)^{2} R^{4 a-2}, \\
r_{2}= & c_{3}^{3} c_{7}^{5} R^{6}\left(S_{4}\right)\left(S_{8}\right), r_{3}=c_{3}^{1} c_{7}^{3} R^{6}\left(S_{4}\right)\left(S_{8}\right), r_{4}=c_{7}^{3} c_{7}^{5} R^{6}\left(S_{8}\right)^{2}, \\
g(a)= & -(2 a-1)\left(R^{2} c_{4 a}^{2 a} S_{4 a}-c_{4 a-2}^{2 a-1} T_{4 a-1}\right), g_{1}=-6 S_{4} R^{2}, \\
g_{2}= & -R^{2} c_{8}^{2} S_{8}+c_{6}^{5} T_{7}, g_{3}=-5 R^{2} c_{8}^{6} S_{8}+5 c_{6}^{5} T_{7}, \\
g_{4}= & -5 R^{2} c_{12}^{6} S_{12}+5 c_{10}^{5} T_{11},
\end{aligned}
$$

where,

$$
\begin{aligned}
\kappa_{\beta}(s) & =\frac{1+2 m_{\beta}}{k_{\beta}},(\beta=1,2), \chi_{m}(s)=\frac{m_{2}}{m_{1}}, \chi_{p}(s)=\frac{p_{1}-p_{2}}{p_{1}+p_{2}}, \\
A(s) & =\left(\kappa_{1} \chi_{m}-\kappa_{2}\right) B(s) /\left(\kappa_{2}+\chi_{m}\right), B(s)=\left(1-\chi_{m}\right) /\left(1+\kappa_{1} \chi_{m}\right), \\
C(s) & =\frac{1}{1+\left(k_{1} V_{2}+k_{2} V_{1}\right) / m_{1}}, D(s)=2\left(\frac{k_{2}}{k_{1}}-1\right) C(s), \\
c_{k}^{l} & =\frac{k !}{l !(k-l) !} .
\end{aligned}
$$

Replacing $a=2$ (or $a=3$ ) into the above formulae the effective relaxation viscoelastic properties $\hat{R}_{11}=\hat{k}+\hat{m}^{\prime}, \hat{R}_{12}=\hat{k}-\hat{m}^{\prime}, \hat{R}_{13}=$ $R_{23}=\hat{l}, \hat{R}_{33}=\hat{n}, \hat{R}_{44}=\hat{p}, \hat{R}_{66}=\hat{m}$ in the Laplace-Carson space of two phase fibrous composites with a square (or hexagonal) distribution of fibres can be computed. The corresponding lattice sums for square $(a=2)$ are $S_{4}=3.1512120, S_{8}=4.2557731, S_{12}=$ $3.9388490, T_{7}=4.5155155, T_{11}=3.8807309, S_{6}=T_{5}=0$; and for an hexagonal array $(a=3) S_{6}=5.8630316, S_{12}=6.00096399, T_{5}=$ 5.6568027, $T_{11}=6.0301854, S_{4}=S_{8}=T_{7}=0$. The infinite system (27)-(29) is used such that it is truncated for obtaining an $n \times n$ order system. It is interesting to note that the effective properties are monotonic functions of order $n$ of the solution of the system. In general, the numerical results converge well to the exact solutions when an adequate order in the solution of the system is chosen as $n$ increases. The truncation order for solving the system increases as the parameters and the fiber volume fraction are high. In the numerical examples the solutions are given for $n=1$, because this order of $n$ achieves the required accuracy for the used parameters. An analysis of different truncation order of the system is carried out in Bravo-Castillero et al. (2012).

\section{Numerical results}

The above described formulae allow to find the value of the effective properties in the time space using the Laplace-Carson in- 
Table 1

Comparison between AHM and (Luciano and Barbero, 1994) for the elastic limit case.

\begin{tabular}{|c|c|c|c|c|c|c|}
\hline \multirow[b]{2}{*}{$V_{2}$} & \multicolumn{2}{|l|}{$R_{11}^{(*)}(\mathrm{Gpa})$} & \multicolumn{2}{|l|}{$R_{12}^{(*)}(\mathrm{Gpa})$} & \multicolumn{2}{|l|}{$R_{13}^{(*)}(\mathrm{Gpa})$} \\
\hline & AHM & Luciano and Barbero (1994) & AHM & Luciano and Barbero (1994) & AHM & Luciano and Barbero (1994) \\
\hline 0.1 & 6.936594879 & 6.945135868 & 4.114809778 & 4.118314686 & 3.976769783 & 3.979069602 \\
\hline 0.2 & 7.985585572 & 8.005989411 & 4.495272042 & 4.503534289 & 4.249682969 & 4.255155939 \\
\hline 0.3 & 9.369227804 & 9.400964062 & 4.885181938 & 4.900928021 & 4.588292058 & 4.597357453 \\
\hline 0.4 & 11.25076105 & 11.26778928 & 5.27452862 & 5.311466277 & 5.021851675 & 5.03215492 \\
\hline 0.5 & 13.9198188 & 13.78735459 & 5.66188142 & 5.778415812 & 5.605385914 & 5.602344571 \\
\hline \multirow[t]{2}{*}{0.6} & 17.96594014 & 17.16219628 & 6.114763016 & 6.486650719 & 6.464341937 & 6.381891347 \\
\hline & \multicolumn{2}{|l|}{$R_{33}^{(*)}(\mathrm{Gpa})$} & \multicolumn{2}{|l|}{$R_{44}^{(*)}(\mathrm{Gpa})$} & \multicolumn{2}{|l|}{$R_{66}^{(*)}(\mathrm{Gpa})$} \\
\hline$V_{2}$ & $\mathrm{AHM}$ & Luciano and Barbero (1994) & AHM & Luciano and Barbero (1994) & AHM & Luciano and Barbero (1994) \\
\hline 0.1 & 12.6838726 & 12.68475077 & 1.424840615 & 1.427492938 & 1.370697667 & 1.372776844 \\
\hline 0.2 & 19.26465085 & 19.26674067 & 1.71926491 & 1.723652839 & 1.567873934 & 1.570729617 \\
\hline 0.3 & 25.87051461 & 25.87397617 & 2.090516656 & 2.094394163 & 1.794030412 & 1.796104297 \\
\hline 0.4 & 32.51263455 & 32.51656877 & 2.578777628 & 2.573622151 & 2.077515799 & 2.074583565 \\
\hline 0.5 & 39.21202123 & 39.21085991 & 3.264965853 & 3.219412229 & 2.472442658 & 2.451878559 \\
\hline 0.6 & 46.01657574 & 45.98509257 & 4.339418865 & 4.14022079 & 3.106691102 & 3.025649366 \\
\hline
\end{tabular}

verse transform, which is denoted by $R_{i j}^{(*)}$ for two-phase viscoelastic medium with isotropic constituents for both types of cell. In the literature, there are different relaxation kernels that describe viscoelastic properties of materials. In this section, different viscoelastic models depending of the kernel types are analyzed. For instance, Maxwell, Kelvin and Rabotnov kernels are used in the computational algorithm for computing the effective properties.

\subsection{Validation of the present model}

The limit case when $t=0$ yields the elastic behavior for the composites. In Table 1 , the comparison of the transversally isotropic elastic effective properties $R_{11}^{(*)}, R_{12}^{(*)}, R_{13}^{(*)}, R_{33}^{(*)}, R_{44}^{(*)}, R_{66}^{(*)}$ between the present model with square cell and (Luciano and Barbero, 1994) are shown. The elastic material parameters used in the computation are $E^{(m)}=3.27 \mathrm{Gpa}$ (Young modulus matrix),
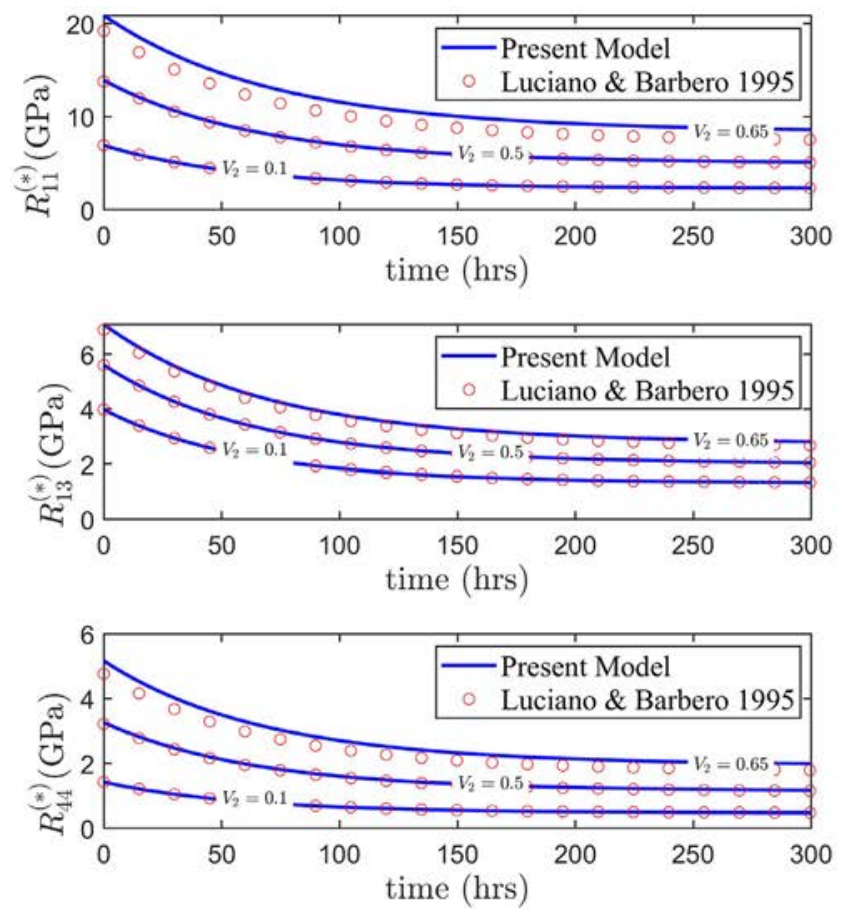

$v^{(m)}=0.38$ (matrix Poisson ratio), $E^{(f)}=68.67 \mathrm{Gpa}$ (Young modulus fibre), and $v^{(f)}=0.21$ (Poisson ratio fibre). Both methods exhibit good agreement for the whole fibre volume fraction range.

\subsection{Numerical behavior of the relaxation moduli}

(1) Figs. 2-4 show the behavior of the relaxation moduli computed by asymptotic homogenization method (AHM) using the formulae (27)-(29).

Comparisons for the whole set of relaxation effective components between the present model via AHM with the results reported in Barbero and Luciano (1995) for a composite with elastic circular fibres embbeded in a viscoelastic matrix are displayed in Fig. 2. The input material parameters are $E^{(f)}=68.67 \mathrm{Gpa}$ (fibre Young modulus), $v^{(f)}=0.21$ (fibre Poisson ratio), and the four-parameter model, involving Maxwell element and KelvinVoight element connected in series (Mainardi and Spada, 2011)
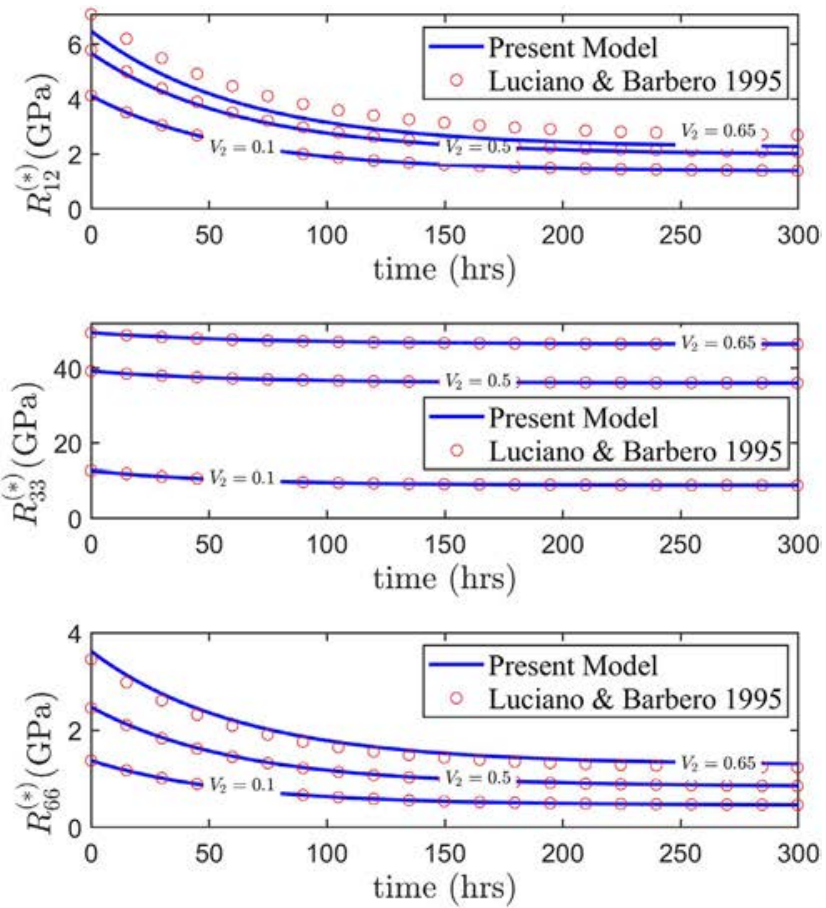

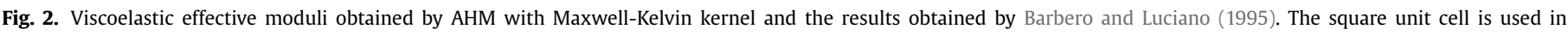
the computation. 

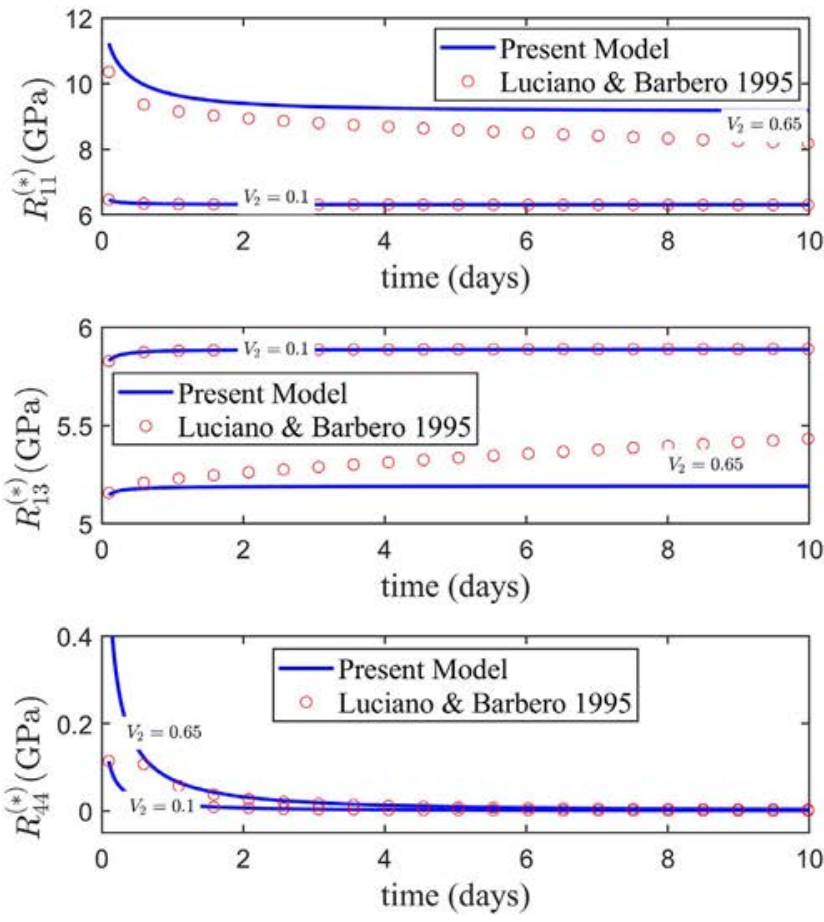
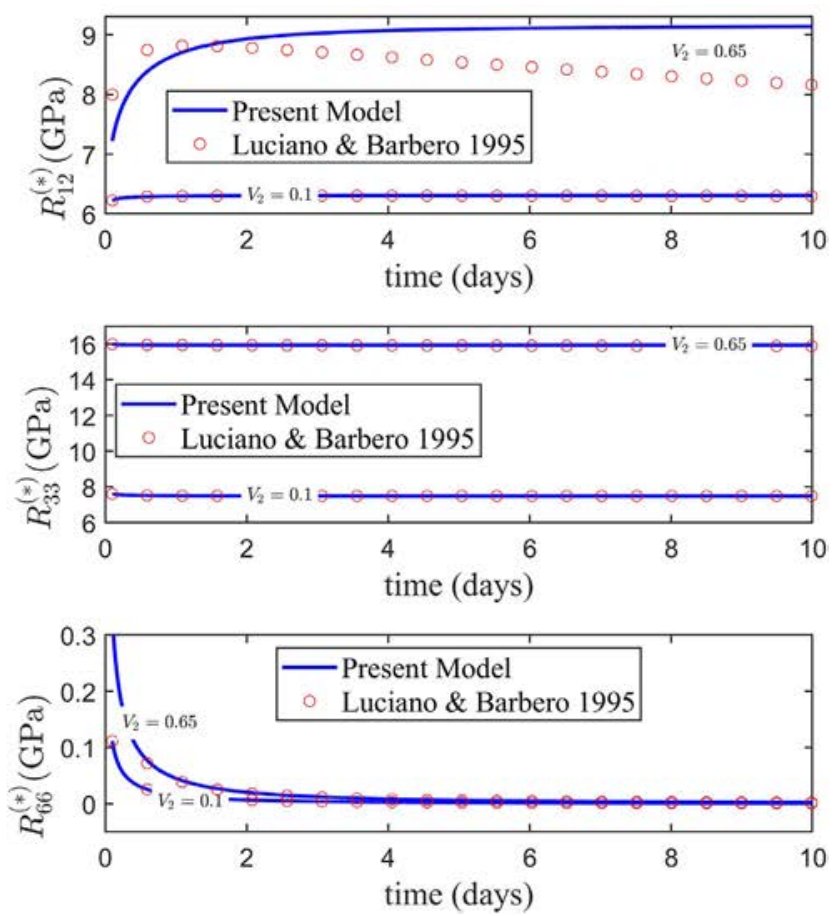

Fig. 3. Comparison between the effective moduli obtained by AHM with (Barbero and Luciano, 1995) for the Rabotnov kernel and square cell.
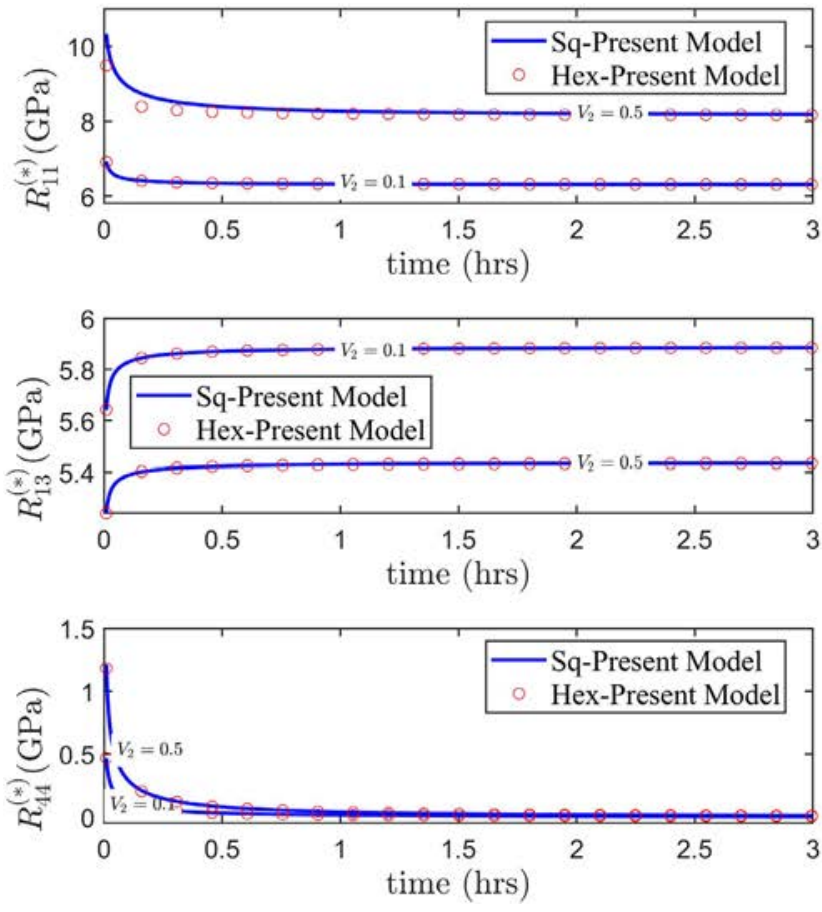
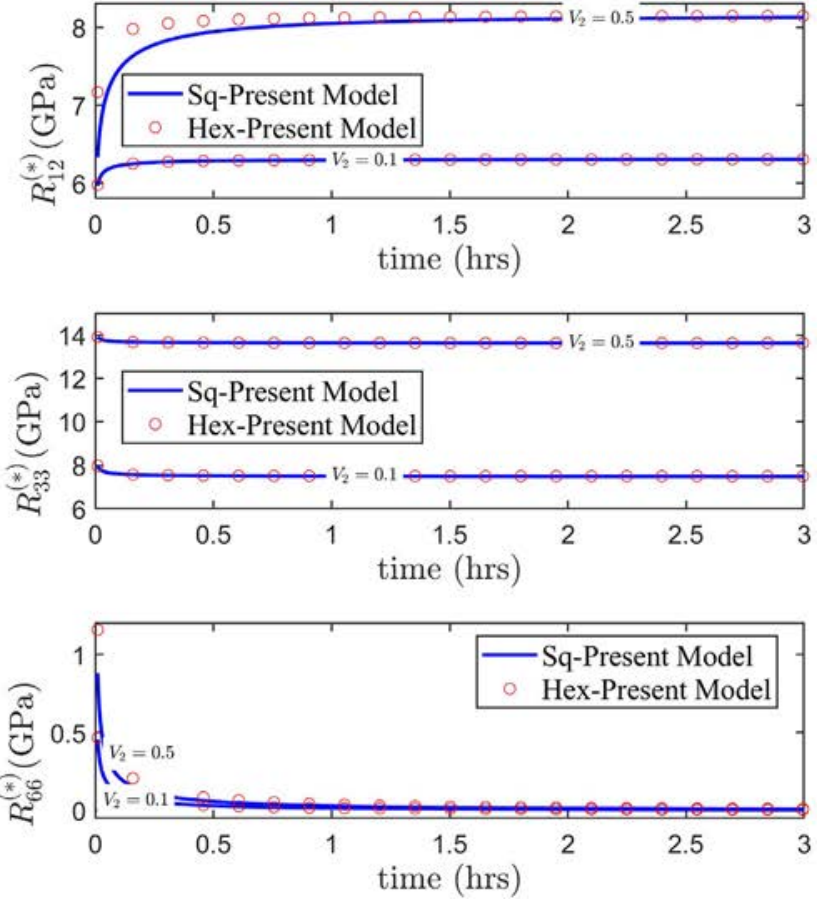

Fig. 4. Effective moduli obtained by AHM using Rabotnov kernel for both (hexagonal and square) cells.

is used for the matrix viscoelastic Young modulus following the analysis reported in Barbero and Luciano (1995) (see, formulae (49)) $\hat{E}^{(m)}(s)=\frac{E^{e} \eta^{M}\left(E^{V}+\eta^{V} s\right) s}{E^{e} E^{V}+\left(E^{V} \eta^{M}+E^{e}\left(\eta^{V}+\eta^{M}\right) s+\eta^{V} \eta^{M} s^{2}\right.}$ where $E^{e}=$ $3.27 \mathrm{GPa}, E^{V}=1.8 \mathrm{GPa}, \eta^{M}=8000 \mathrm{GPa} \cdot \mathrm{hr}, \eta^{V}=300 \mathrm{GPa} \cdot \mathrm{hr}$, and $v^{(m)}=0.38$ (matrix Poisson ratio). In Fig. 2, the effective coefficients for the Maxwell-Kelvin kernel (see Zhang and OstojaStarzewski, 2015) are shown with different volume fractions, i.e., $V_{2}=0.1, V_{2}=0.5$ and $V_{2}=0.65$. We can observe a good agree- ment between both approaches (AHM and the results reported in Barbero and Luciano, 1995). The models practically coincide for small volume fractions and exhibit slight difference for higher volume fractions.

(2) Nowadays, the fractional exponential function $\ni_{\alpha}(\beta, t)$ introduced by Rabotnov (see Rabotnov, 1948) as $\ni_{\alpha}(\beta, t-\tau)=(t-$ $\tau)^{\alpha} \sum_{n=0}^{\infty} \frac{(\beta)^{n}(t-\tau)^{n(1+\alpha)}}{\Gamma[(n+1)(1+\alpha)]}$, is also known as the Rabotnov function and as a special case of the Mittag-Leffler function widely used in 

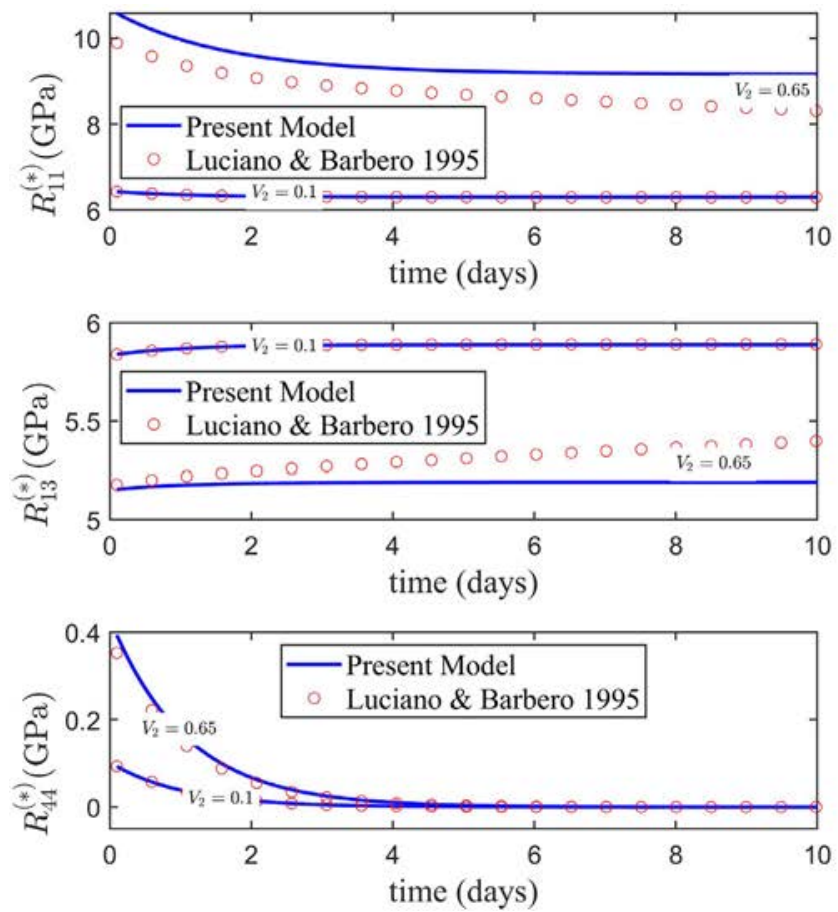
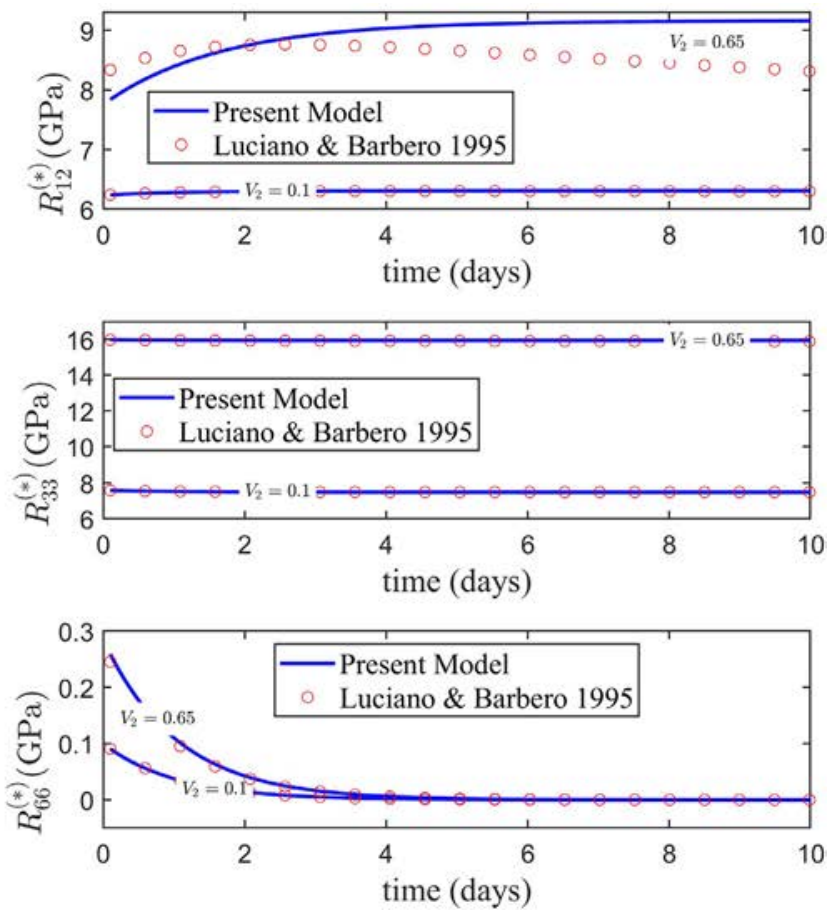

Fig. 5. Comparison of the effective moduli obtained by AHM with Barbero and Luciano (1995) for the Kelvin kernel. The square unit cell is used in the computation.
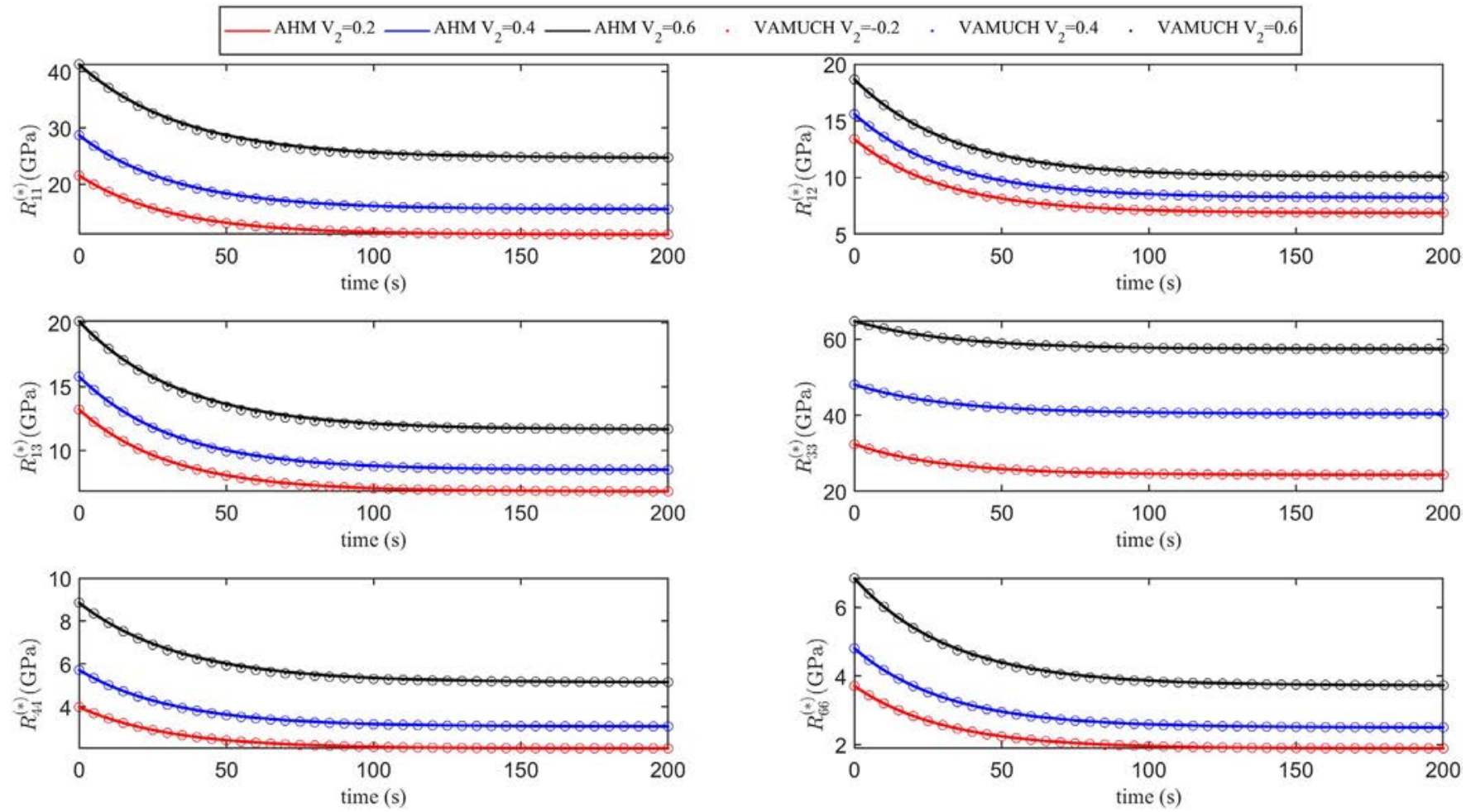

Fig. 6. Comparison of the effective moduli obtained by AHM with VAMUCH (Tang and Felicelli, 2015) using Prony series. The square unit cell is used in the computation.

fractional calculus (Rossikhin and Shitikova, 2014). In this sense, Fig. 3 presents the computation of $R_{11}^{(*)}, R_{12}^{(*)}, R_{13}^{(*)}, R_{33}^{(*)}, R_{44}^{(*)}$, $R_{66}^{(*)}$ relaxation effective properties using both models (AHM and Barbero and Luciano, 1995) with Rabotnov kernel (see, Rabotnov, 1948, Rabotnov, 1977) and $V_{2}=0.1, V_{2}=0.65$ volume fractions. The material parameters used in the computation are: elastic fibre $\left(\mu^{(f)}=8.571 \times 10^{9}, K^{(f)}=10.0 \times 10^{9}\right)$ and viscoelastic matrix $\left(\alpha^{(m)}=0.47, \quad \beta^{(m)}=0.98, \quad \lambda^{(m)}=49.6, \quad \mu_{0}^{(m)}=1.7 \times 10^{6}, K^{(m)}=\right.$ $\left.5.97 \times 10^{9}\right)$, and $\mu^{(m)}=s \cdot \mu_{0}^{(m)} \cdot\left(1+\frac{\lambda^{(m)}}{s^{\left(1-\alpha^{(m)}\right.}+\beta^{(m)}}\right)$. Notice that for $V_{2}=0.1$ volume fraction all the effective properties coincide whereas for higher volume fraction, for instance, $V_{2}=0.65$, there are differences for $R_{11}^{(*)}, R_{12}^{(*)}, R_{13}^{(*)}$ overall coefficients, however the coefficients exhibit the same tendency. 
Besides, Fig. 4 shows the viscoelastic effective properties for circular fibres with hexagonal and square cells using Rabotnov kernel for two volume fractions $V_{2}=0.1$ and $V_{2}=0.5$. The material parameters used are the same to the previous figure. It is worth remarking that the effective coefficients $R_{11}^{(*)}, R_{12}^{(*)}, R_{66}^{(*)}$ for $V_{2}=0.5$ volume fraction exhibit a difference between square and hexagonal cells for low values of time whereas for $V_{2}=0.1$ volume fraction the curves do not show difference in the whole time range. Moreover, $R_{13}^{(*)}, R_{33}^{(*)}, R_{44}^{(*)}$ for square and hexagonal cells behave in a similar form and they coincide in the whole time interval.

In addition, as a particular case of Rabotnov model for $\alpha=0$, Fig. 5 displays the numerical simulation between the two aforementioned approaches for the Kelvin kernel with volume fraction $V_{2}=0.1$, and $V_{2}=0.65$. Notice the remarkable difference in the effective coefficients $R_{11}^{(*)}, R_{12}^{(*)}, R_{13}^{(*)}$, for high volume fraction. A possible source that contributes to the discrepancy for high volume fraction may be related to the fact that the systems are truncated to the second order. However, the obtention of unified analytical expressions for different geometries justifies the procedure followed in the model.

(3) Recently, (Tang and Felicelli, 2015) reports a micromechanic model to characterize the effective stress relaxation stiffness of polymer composites. The linear viscoelastic behavior of polymer material is modeled by hereditary integral. The proposed model is established based on the variational asymptotic method for unit cell homogenization (VAMUCH). The computations using this model is done in the time domain, where the Laplace transform and inversion commonly used for linear viscoelastic composites are not needed. The accuracy and efficiency of the proposed model is verified by comparing with the results of Representative Volume Element Model developed using ABAQUS. The present model is validated with the efficient approach VAMUCH for different fibre volume fraction and the results are displayed in Fig. 6. The fibres direction $x_{1}$ reported by Tang and Felicelli (2015) has been interchanged to $x_{3}$ in AHM. The material parameters used (Tang and Felicelli, 2015) are: for the elastic fibre $E^{(f)}=80 \mathrm{Gpa}$ (Young modulus) and $v^{(f)}=0.3$ (Poisson ratio); and the viscoelastic matrix using Prony series as $E^{(m)}(t)=0.5 E_{0}^{(m)}(1+\exp (-t / 30))$ (Young modulus) where $E_{0}^{(m)}=8 \mathrm{Gpa}$ and $v^{(m)}=0.4$ (Poisson ratio).

\section{Conclusions}

In this work, the two-scale asymptotic homogenization method is applied to calculate the linear viscoelastic effective properties for fibrous composites where the distribution of the fibres have a preferential axis. The local problems and the analytical expressions of the effective relaxation coefficients are obtained for square and hexagonal cells as an extension of previous works (see GuinovartDíaz et al., 2001; Rodríguez-Ramos et al., 2001), where the overall properties were derived for fibrous elastic composites. In addition, the influence of different viscoelastic models on the relaxation effective behavior is considered, in particular, the Maxwell-Kelvin model, Rabotnov's fractional exponential kernel and Kelvin model are analyzed. Numerical computations of Laplace-Carson's inverse are implemented for the calculation of the effective viscoelastic relaxation properties. Comparisons with other approaches show the efficiency of the method and it provides a good benchmark with theoretical and experimental models.

Also, this work establishes new perspectives for further investigations; for instance, the results can be extended to the case of imperfect contact conditions by following the methodology in Otero et al. (2016) and considering viscoelastic interfaces (see Daridon et al., 2016). Another natural step is to generalize the present work to ageing viscoelastic solids (see Sanahuja, 2013).

\section{Declaration of Competing Interest}

The authors declare that they have no known competing financial interests or personal relationships that could have appeared to influence the work reported in this paper.

\section{Acknowledgements}

The authors would like to thank the Basic Science Department of the Institute Technological of Monterrey, Campus of Mexico State for its support. France-Cuba project "Partenariat Hubert Curien franco-cubain Carlos J. Finlay" 2017-2018 and the funding of Proyecto Nacional de Ciencias Básicas (MATCOM) 2019, Universidad de La Habana are gratefully acknowledged. Thanks to Departamento de Matemáticas y Mecánica IIMAS-UNAM and PREIDGAPA-UNAM for their support and Ramiro Chávez Tovar and Ana Pérez Arteaga for computational assistance. OLCG kindly thanks to Ecole Doctorale No. 353 de L'Université de Aix Marseille and L'équipe Matériaux \& Structures du Laboratoire de Mécanique et d'Acoustique LMA - UMR 7031 AMU - CNRS - Centrale Marseille 4 impasse Nikola Tesla CS 4000613453 Marseille Cedex 13, France. The authors thank Prof. Tian Tang of the University of Akron, United States for providing the variational asymptotic method for unit cell homogenization (VAMUCH) data employed in the comparison of Fig. 6. JB and FJS acknolwedges the funding of PAPITDGAPA-UNAM IA100919. RGD and RRR would like to thank to COIC/STIA/ 9042 and COIC/STIA/9045/2019.

\section{References}

Bakhvalov, N.S., Panasenko, G. 1989. Homogenisation: averaging processes in periodic media: mathematical problems in the mechanics of composite materials. Mathematics and its Applications. Kluwer Academic Publishers.

Barbero, E.J., Luciano, R., 1995. Micromechanical formulas for the relaxation tensor of linear viscoelastic composites with transversely isotropic fibers. Int. J. Solids Struct. 32 (13), 1859-1872.

Bravo-Castillero, J., Guinovart-Díaz, R., Rodríguez-Ramos, R., Sabina, F., Brenner, R., 2012. Unified analytical formulae for the effective properties of periodic fibrous composites. Mater. Lett. 73, 68-71.

Brenner, R., Masson, R., Castelnau, O., Zaoui, A., 2002. A quasi-elastic affine formulation for the homogenized behavior of nonlinear viscoelastic polycristals and composites. Eur. J. Mech. A/ Solids 21, 943-960.

Cai, Y., Sun, H., 2013. Prediction on viscoelastic properties of three-dimensionally braided composites by multi-scale model. J. Mater. Science 48, 6499-6508.

Christensen, R.M., 1982. Theory of Viscoelasticity - 2nd Edition An Introduction. Academic Press

Daridon, L., Licht, C., Orankitjaroen, S., Pagano, S., 2016. Periodic homogenization for Kelvin-Voigt viscoelastic media with a Kelvin-Voigt viscoelastic interphase. Eur. J. Mech. A Solids 58, 163-171.

Guinovart-Díaz, R., Bravo-Castillero, J., Rodríguez-Ramos, R., Sabina, F., 2001. Closedform expressions for the effective coefficients of fibre-reinforced composite with transversely isotropic constituents. i: elastic and hexagonal symmetry. J Mech Phys Solids 49, 1445-1462.

Hashin, Z., 1966. Viscoelastic fibre reinforced materials. AIAA J. 4, 1411-1417

Hollenbeck, K., 1998. INVLAP.M: a Matlab function for numerical inversion of laplace transforms by the Hoog algorithm, URL https://www.mathworks.com/ matlabcentral/answers/uploaded_files/1034/invlap.m.

Hu, R., Oskay, C., 2018. Spatial-temporal nonlocal homogenization model for transient anti-plane shear wave propagation in periodic viscoelastic composites. Comput. Methods Appl. Mech. Eng. 342 (1), 1-31

Lahellec, N., Suquet, P., 2007. Effective behavior of linear viscoelastic composites : a time-integration approach. Int. J. Solids Struct. 44 (2), 507-529. doi:10.1016/j. ijsolstr.2006.04.038

Le, Q., Meftah, F., He, Q., Le Pape, Y., 2007. Creep and relaxation functions of a heterogeneous viscoelastic porous medium using the Mori-Tanaka homogenization scheme and a discrete microscopic retardation spectrum. Mech. Time Depend. Mater. 11 (3-4), 309-331.

Lévesque, M., Gilchrist, M., Bouleau, N., Derrien, K., Baptiste, D., 2007. Numerical inversion of the laplace-carson transform applied to homogenization of randomly reinforced linear viscoelastic media. Comput. Mech. 40, 771-789.

Levin, V., Sevostianov, I., 2005. Micromechanical modeling of the effective viscoelastic properties of inhomogeneous materials using fraction exponential operators. Int. J. Fract. 134 (3-4), 37-44.

Liu, S., Chen, K.-Z., Feng, X.-A., 2004. Prediction of viscoelastic property of layered materials. Int. J. Solids Struct. 41, 3675-3688.

Luciano, R., Barbero, E.J., 1994. Formulas for the stiffness of composites with periodic microstructure. Int. J. Solids Struct. 31 (21), 2933-2944. 
Maghous, S., Creus, G.J., 2003. Periodic homogenization in thermoviscoelasticity: case of multilayered media with ageing. Int. J. Solids Struct. 40 (4), 851-870. doi:10.1016/S0020-7683(02)00549-8.

Mainardi, F., Spada, G., 2011. Creep, relaxation and viscosity properties for basic fractional models in rheology. Eur. Phys. J. Spec. Top. 193, 133-160.

Otero, J.A., Rodríguez-Ramos, R., Monsivais, G., 2016. Computation of effective properties in elastic composites under imperfect contact with different inclusion shapes. Math. Methods Appl. Sci. 40 (9), 3290-3310. doi:10.1002/mma.3956.

Persson, L., Persson, L., Svanstedt, N., Wyller, J., 1993. The homogenization method. an introduction.

Pipkin, A.C., 1986. Lectures on Viscoelasticity Theory, second ed. Springer-Verlag.

Pobedria, B., 1984. Mechanics of Composite Materials. Moscow State University Press.

Podlubny, L., 1998. Fractional Differential Equations. Academic Press.

Rabotnov, Y., 1948. Equilibrium of an elastic medium with after-effects. J. Appl. Math. Mech. (PMM) 12, 53-62.

Rabotnov, Y., 1977. Elements of Hereditary Solid Mechanics. Mir.

Rodríguez-Ramos, R., Sabina, F., Guinovart-Díaz, R., Bravo-Castillero, J., 2001. Closedform expressions for the effective coefficients of fibre-reinforced composite with transversely isotropic constituents. i: elastic and square symmetry. Mech. Mat. 33. 223-235

Rossikhin, Y.A., Shitikova, M.V., 2014. The simplest models of viscoelasticity involving fractional derivatives and their connectedness with the Rabotnov fractional order operators. Int. J. Mech. 8, 326-331.

Sabina, F., Bravo-Castillero, J., Guinovart-Díaz, R., R., R.-R., Valdiviezo-Mijangos, O. 2002. Overall behavior of two-dimensional periodic composites. Int. J. Solids Struct. 39(2), 483-497.

Sanahuja, J., 2013. Effective behaviour of ageing linear viscoelastic composites: homogenization approach. Int. J. Solids Struct. 50 (19), 2846-2856.

Schapery, R., 1964. Application of thermodynamics to thermomechanical, fracture, and birefringent phenomena in viscoelastic media. J. Appl. Phys. $35,1451-1465$.

Scott Blair, G.W., Coppen, F.M.V., 1939. The subjective judgement of the elastic and plastic properties of soft bodies: the differential thresholds for viscosities and compression moduli. Proc. R. Soc. B 128, 109-125.
Scott Blair, G.W., Coppen, F.M.V., 1943. The estimation of firmness in soft materials. Am. J. Psychol. 56, 234-246.

Sevostianov, I., Levin, V., 2015. Creep and relaxation contribution tensors for spheroidal pores in hereditary solids: fraction-exponential operators approach Acta Mechanica 227(1), 217-227.

Sevostianov, I., Levin, V., Radi, E., 2016. Effective viscoelastic properties of short-fiber reinforced composites. Int. J. Eng. Sci. 100, 61-73.

Shermergor, T., 1977. Theory of Elasticity for Micro-Heterogeneous Media. Nauka.

Tang, T., Felicelli, S.D., 2015. Computational evaluation of effective stress relaxation behavior of polymer composites. Int. J. Eng. Sci. 90, 76-85.

To, Q.-D., Nguyen, S.-T., Bonnet, G., Vu, M.-N., 2017. Overall viscoelastic properties of $2 \mathrm{~d}$ and two-phase periodic composites constituted of elliptical and rectangular heterogeneities. Eur. J. Mech. A Solids doi:10.1016/j.euromechsol.2017.03.004.

Tran, A., Yvonnet, J., He, Q.-C., Toulemonde, C., Sanahuja, J., 2011. A simple computational homogenization method for structures made of linear heterogeneous viscoelastic materials. Comput. Methods Appl. Mech. Eng. 200, 2956-2970.

Tressou, B., Vaziri, R., Nadot-Martin, C., 2018. Application of the incremental variational approach (eiv model) to the linear viscoelastic homogenization of different types of microstructures: long fiber,-particle reinforced and strand-based composites. Eur. J. Mech. A Solids doi:10.1016/j.euromechsol.2017.10.006.

Valdivieso-Mijangos, O., Guinovart-Díaz, R., Bravo-Castillero, J., Sabina, F., Rodríguez-Ramos, R., 2002. Elastic effective coefficients of fiber-reinforced composite with cubic symmetry constituents in a hexagonal arrangement. Mech. Res. Commun. 29, 113-119.

Vilchevskaya, E., Levin, V., Seyedkavoosi, S., Sevostianov, I., 2019. Replacement relations for a viscoelastic material containing multiple inhomogeneities. Int. J. Eng. Sci. 136, 26-37.

Volterra, V., 1912. Sur les équations intégro-différentielles et leurs applications. Acta Mathematica 35, 295-356.

Wang, G., Pindera, M.-J., 2016. Locally-exact homogenization of viscoelastic unidirectional composites. Mech. Mater. 103, 95-109.

Yi, Y.-M., Park, S.-H., Youn, S.-K., 1998. Asymptotic homogenization of viscoelastic composites with periodic microstructures. Int. J. Solids Struct. 35 (17) 2039-2055.

Zhang, J., Ostoja-Starzewski, M., 2015. Mesoscale bounds in viscoelasticity of random composites. Mech. Res. Commun. 68, 98-104. 